\section{Redacción publicitaria y redes sociales: el desafío de la globalización}

\author{
Copywriting and social networks: the challenge of globalization
}

\author{
Diana Anzuátegui Macías
}

\section{RESUMEN}

El siglo XXI y la globalización a la que el mundo ha sido arrastrado, escenifica una sociedad que no puede existir sin las redes sociales, y que a través de ésta se ve enfrentada a una serie de complejidades, entre ellas la publicidad como elemento vital para la transferencia de valor y productos. Con el avance de la tecnología y los medios para el acceso a los distintos productos las redes sociales ocupan un lugar importante para el posicionamiento de los objetos de consumo. El artículo busca reflexionar sobre el desafío de la globalización en la profesión del redactor publicitario y cómo las redes sociales han ayudado a maximizar la complejidad del siglo XXI. Para esto, se realizó un análisis bibliográfico de fuentes especializadas en la temática; enfatizando principalmente el uso de la lengua inglesa como agente transmisor de cultura e ideologías exógenas, en el escándalo del capitalismo de vigilancia que irrumpe en la privacidad de los internautas, y por último en el fenómeno de las redes sociales. Se concluye que la profesión del publicista no es solo una cuestión técnica y creativa en favor de una marca, sino que abarca una responsabilidad ética ante los abusos de las empresas que recopilan datos sin consentimiento, implica un rol importante en la conservación de valores culturales y que deben ser tomados en cuenta con mayor atención.

Palabras clave: publicidad; globalización, consumo; capitalismo vigilancia; siglo XXI

\section{ABSTRACT}

The 21st century and the globalization to which the world has been dragged into, has created a society that cannot exist without social networks, and that through it is confronted with a series of complexities, including advertising as a vital element for the transfer of value and products. With the advance of technology and the means of access to different products, social networks occupy an important place in the positioning of consumer objects. The article seeks to reflect on the challenge of globalization in the copywriting profession and how social networks have helped to maximize the complexity of the 21st century. For this, a bibliographic analysis of specialized sources on the subject was carried out, emphasizing mainly the use of the English language as a transmitting agent of culture and exogenous ideologies, the scandal of surveillance capitalism that bursts into the privacy of Internet users, and finally the phenomenon of social networks. It is concluded that the profession of the publicist is not only a technical and creative issue in favor of a brand, but also encompasses an ethical responsibility in the face of abuses by companies that collect data without consent, implies an important role in the preservation of cultural values and should be taken into account with greater attention.

Keywords: advertising; globalization, consumption; surveillance capitalism; 21st century
RELIGACIÓN

REVISTA DE CIENCIAS SOCIALES Y HUMANIDADES JOURNAL OF SOCIAL SCIENCES AND HUMANITIES REVISTA DE CENCIAS SOCIAIS E HUMANAS

INFORMACIÓN:

https://doi.org/10.46652/rgn.v6i30.872

ISSN $2477-9083$

Vol. 6 No. 30, 2021. e210872

Quito, Ecuador

Enviado: agosto 03, 2021

Aceptado: octubre 15, 2021

Publicado: noviembre 01, 2021

Publicación Continua

Sección Sur-Sur | Peer Reviewed

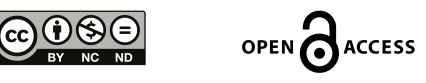

AUTORA:

Diana Anzuátequi Macías Voronezh States University -

Federación Rusa

danzuategui@vsu.ru

CONFLICTO DE INTERESES

La autora declara que no existe conflicto de interés posible.

FINANCIAMIENTO

No existió asistencia financiera de partes externas al presente artículo. AGRADECIMIENTO

$\mathrm{N} / \mathrm{A}$

NOTA

El artículo no se desprende de un trabajo anterior.

ENTIDAD EDITORA 


\section{Introducción}

La volatilidad de los tiempos, el desarrollo de la ciencia y la tecnología han permitido la modificación de las formas de vida, consumo, organización social y política de la sociedad mundial. Los medios y mecanismos empleados en siglos anteriores difieren en gran manera a los empleados en el presente siglo, cambios que son profundizados por los fenómenos de la globalización y la irrupción de las redes sociales. El campo de la publicidad también se ha visto afectada por estos cambios; es así que redactar anuncios publicitarios durante la revolución industrial, o mediante el surgimiento de la radio, o el cine, se han transformado en la actualidad, sobre todo con el aparecimiento de las redes sociales y su propia evolución y competencia entre aplicaciones que inundan de datos a los potenciales consumidores.

Con el rápido avance de la tecnología e internet, la web 2.o han llegado a ocupar un lugar importante y hasta decisivo en el presente siglo. Las redes sociales han planteado amenazas y oportunidades para la publicidad (Jung, 2015), según Liu (2013) en su estudio sobre la influencia de la publicidad en redes sociales, encontró que más de la mitad de los estudiantes están influenciados por las redes sociales cuando compran algo en línea. El entorno virtual se ha vuelto totalmente interactivo y colaborativo, con individuos que participan activamente en la comunicación bidireccional, que publican reseñas de servicios y productos, suben vídeos creados por ellos mismos e incluso viven vidas virtuales.

La gran exposición que viven los usuarios de las Tecnologías de la información y la comunicación - TIC y redes sociales, ha hecho que éstas sirvan en casi todos los campos de la vida, para comunicarse, buscar información, comprar, aprender, vender, conocer personas dentro y fuera de sus países, etc., (Chetioui et al., 2020; Cordero-Gutierrez \& Lahuerta-Otero, 2020).

Esta nueva época enfrenta al publicista a desafíos que no se presentaban en el pasado siglo. Zuboff (2019) entiende que estamos viviendo la era del capitalismo de vigilancia, mismo que mercantiliza la experiencia humana que es ahora materia prima libre, que se transforma en datos de comportamiento y que luego se vende como "productos de predicción". Aquí las redes sociales cumplen el rol vigilante que compromete de manera ética al rol de publicista pues está enfrentado a la sociedad de consumo, y junto a Rey (2007, p. 158), se puede decir que son "malos tiempos para la ética, cuando el individualismo recorre el planeta. Malos tiempos para la honestidad, cuando el materialismo inunda el mundo". Esto se ve ejemplificado, en las grandes sumas de dinero que obtienen las redes sociales como producto de la venta de publicidad, según Marimar Jiménez y Álvaro Bayón (2021), solo en 2020 la red social Facebook obtuvo ganancias netas por 29.146 millones de dólares.

Por otra parte, la globalización trae consigo la unificación o la masificación del uso de una lengua, el inglés. Junto con la difusión de la lengua, la cultura también ha ido tomando lugar y modificando las culturas y lenguas locales. Tal como lo recoge Beltrán y Cruces “toda deformación del lenguaje, toda adopción de extranjerismos y de modismos vulgares, todo descuido ortográfico publicado en los anuncios, tarde o temprano distorsiona la cultura de los pueblos" (2006, p. 19). Por lo tanto, no sólo se trata de cómo redactar mejores anuncios para los publicistas, sino que tras sus anuncios están consolidad una u otra cultura.

Los profesionales de la publicidad han reconocido el impacto cultural y económico de los medios sociales y se han esforzado por reaccionar ante el cambio resultante, por lo tanto, este artículo tiene como fin abordar las reflexiones sobre los desafíos del quehacer publicitario ante el fenómeno de las redes sociales en el siglo XXI. 


\section{Metodología}

Se utilizó un análisis documental con base en artículos académicos procedentes de bases de datos importantes como Scopus, Communication \& Mass Media Index, WoS, de revistas especializadas en Marketing y Publicidad, así como libros de autores relevantes en cuanto a sistematización de la historia de la publicidad que posibilitaron el análisis de campos específicos que responden al desafío del rol del publicista ante las redes sociales. Para esto se escogieron los ámbitos de la cultura popular y la sociedad de consumo, la globalización, el uso del lenguaje, la potencialidad de las redes sociales, como elementos que condicionan/motivan el rol del publicista en el presente siglo.

\section{Desarrollo}

\subsection{Tiempos inquietantes. Publicidad y sociedad de consumo}

Como consecuencia de la modernización, las sociedades han experimentado diversos cambios que configuran también nuevos comportamientos y valores. Los valores tradicionales vinculados a la autoridad y al deber son sustituidos por la autorrealización y los valores individualistas (Rinallo et al., 2016). El despunte industrial y económico ha permitido una mayor circulación de capital y por ende mayor dinero a disposición para el consumo de productos. Los objetos en la cultura de consumo ocupan un lugar destacado en las teorías del yo consumidor, las identidades de los consumidores y las comunidades de consumidores (Belk 1988; Holt 1998; Kleine et al., 1995; Kozinets 2001). Los estudios sociológicos y antropológicos han puesto de manifiesto los rituales, a menudo idiosincrásicos, que utilizan los consumidores para apropiarse de los objetos, desmercantilizarlos y singularizarlos (Belk et al., 1989) y los numerosos e intrincados procesos que conducen al apego psicológico y emocional a los objetos de consumo (Belk 1992).

Los consumidores se implican mucho y de forma duradera con los objetos que les despiertan interesan y les estimulan. Se convierten en ansiosos coleccionistas y miembros de tribus de consumo centradas en los objetos (Baudrillard 1994; Benjamin 1968; Maffesoli 1996; Schouten \& McAlexander 1995). Por su parte, Cova (1997) y Kozinets (1999) sostienen que los objetos crean y mantienen relaciones entre el individuo y la comunidad, mientras que los teóricos sociales y culturales han debatido el papel crucial de los objetos, en particular los objetos comerciales asignados por el mercado, en la (re)producción de las relaciones de clase, género y raza (Bourdieu 1984; Comaroff 1996).

Bajo esta sociedad que genera deseos, el publicista tiene en frente suyo, las condiciones y medios suficientes para ejercer ese rol que posibilita el acercamiento entre objetos y sujetos, entre mercancía y consumidores. En un mundo que, a pesar de los esfuerzos por la lucha contra el cambio climático, todavía mantiene en su pensamiento que los recursos del planeta son infinitos, pues esto se explica en el aumento de productos en el mercado, la sobreproducción que debido a alta demanda siguen consumiendo los recursos del planeta. Lo antes expuesto, explica precisamente el por qué el fundamento de la esta sociedad está basado en el deseo, y que a su vez es un factor que remarca las condiciones de clase, género, es decir una segmentación de la que el publicista toma nota para afirmar estas diferenciaciones; por un lado, irán las campañas, estilos de redacción publicitarios para los estratos más altos de la sociedad y otro camino tomarán aquellas destinadas para segmentos de los quintiles más bajos.

El campo de la publicidad en la presente era, no abarca únicamente objetos sino también que se ha convertido en el medio para conseguir comportamientos específicos. Es común que métodos 
de marketing y publicidad estén siendo aplicados a la religión, a la comunicación política, que también se posicionan como productos, despojando el trasfondo ideológico, históricos de estos, en la misma línea del discurso del final de la historia, dejando los meta relatos de lado, al punto que, a nivel individual de la sociedad de consumo religiosa, los individuos pueden experimentar la sacralidad al comprar y consumir (Belk et al., 1989). En estas condiciones, el componente ético también debe ser tomado en cuenta, pues el publicista tiene en sus manos toda la información que necesita para sugestionar, motivar a los consumidores, quienes no cuestionan el entorno virtual y son bombardeados de información, imágenes, sonidos con los que terminan capitulando y cerrando el ciclo del objetivo del producto.

\subsection{Lenguaje y la globalización. La cultura bajo ataque o defensa de la publicidad}

Es un hecho conocido que la globalización es un proceso de unificación del mundo entero al tener los mismos bienes, estilos y tecnologías. La globalización debe considerarse no sólo como una forma de hacer negocios o de dirigir los mercados financieros, sino que se convirtió en un proceso (Isakova, 2021). Por ejemplo, "el sector de servicios británico trata con sus clientes a través de un centro de llamadas en la India, o un fabricante diseña sus productos en Europa o en el sudeste asiático y los vende en Norteamérica" (p. 3). Hay varios ejemplos en los que el Reino Unido y Estados Unidos han tenido una gran influencia en la economía, la política y la cultura mundial, promoviendo sus iniciativas, movimientos, modos de vida y tecnologías.

Dentro de los elementos que se han masificado y permitido la consolidación de la globalización se encuentra la lengua. El uso del idioma inglés se ha impuesto como mecanismo de entendimiento y comunicación en el mundo, por medio de películas, música, documentos legales, etc. DemontHeinrich recoge algunas fundamentaciones que abogan por la hegemonía del idioma inglés:

...como un hecho simple y en gran medida poco problemático del orden global, al tiempo que celebra sus tendencias y capacidades progresivas supuestamente intrínsecas. Entre ellos se encuentran la superioridad del inglés como proveedor de la realidad "objetiva”, su capacidad para facilitar el éxito individual y colectivo (económico), su capacidad para avanzar en la producción y el intercambio de conocimientos e información, y su condición de otorgante de voz y unidad universal (global) (2008, p. 136)

La lengua transmite un pensamiento, una ideología, unas costumbres que también han calado hondo en el mundo entero, sobre todo en Occidente. La educación formal y la reproducción de la cultura hegemónica han afianzado el pensamiento único que incluso trastocan las culturas locales. Aterrizando este panorama al caso de la publicidad donde el influjo de la lengua extranjera es evidente no sólo en los términos empleados en la publicidad, sino que también se observa en cuanto a la formación del publicista, los métodos empleados en la creación de publicidad, pues estos son formados mayormente con la mentalidad norteamericana o inglesa. El sostener esta estructura, como dice Beltrán y Cruces, tarde o temprano se logra distorsiona la cultura de los pueblos (2006). Es este el peligro que enfrenta el redactor publicitario, aupar el uso abusivo o encubridor de la lengua y ahondar la crisis cultural en el siglo XXI o, por el contrario, posibilitar formas locales de hacer comunicación, de hacer publicidad, y es que así "habrá verdadera publicidad latinoamericana cuando aprendamos a pensar en castellano y de acuerdo con nuestros valores culturales" (p. 37).

En la actualidad, el uso abusivo de extranjerismos incluso ha llegado a posicionar nuevos roles comunicativos, de mucha importancia. Ejemplo de esto son los denominados influencers, 
quienes reproducen los mismos mecanismos exógenos de formato e incluso de objetos que se promocionan dentro de América Latina. Desafiar la tendencia global, implicaría deconstruir un sistema de educación y formación generando nuevas mallas curriculares en los centros educativos que gradúen nuevos publicistas con valores y arraigo cultural, que apuesten a la exposición, refuerzo y consolidación de los valores culturales locales, regionales sin dejar de atender a esa visión global que nos gobierna. Además, implicaría un desarrollo más honesto de la profesión, más creativo e interesado en los procesos de construcción locales de la cultura, el consumo y las necesidades de los consumidores/clientes, una mayor identificación con las marcas y objetos de consumo.

\subsection{Redes sociales. El nuevo poder mediático}

La irrupción de las redes sociales rápidamente acompañó el estilo de vida de consumo del silgo $\mathrm{XXI}$, añadiendo componentes mercantiles a las nacientes redes. Facebook, que comenzó como una red para contactar gente del círculo cercano, compañeros de estudios, familia, hoy en día terminó convirtiéndose en uno de los medios más importante para la publicidad y venta de productos y servicios. De ahí que la mayoría de los sectores de la economía se han volcado a las distintas redes para consolidar su presencia en el mercado (Chan \& Kumar, 2017; Crammond et al., 2018; Waters et al., 2009). Como dicen Kaplan \& Haenlein,

Hoy en día, todo gira en torno a los medios sociales. Algunos gurús del sector afirman que, si no se participa en Facebook, YouTube y Second Life, ya no se forma parte del ciberespacio. Los medios sociales permiten a las empresas entablar un contacto oportuno y directo con el consumidor final a un coste relativamente bajo y con un nivel de eficiencia superior al que se puede conseguir con las herramientas de comunicación más tradicionales. Esto hace que los medios sociales no sólo sean relevantes para las grandes empresas multinacionales, sino también para las pequeñas y medianas empresas, e incluso para los organismos gubernamentales y sin ánimo de lucro (2010, p. 67).

En la actualidad, encontramos una mayor exposición a los medios de comunicación de masas y a las redes sociales (televisión, radio, Internet, Facebook, YouTube, twitter, WhatsApp, etc.). Los individuos dedican cada vez más tiempo a exponerse e interactuar con los medios de comunicación de masas y las redes sociales (Jung, 2015).

Esta sobre exposición no es gratuita, así el director de gestión de producto de Facebook ha reconocido que la compañía estadounidense utiliza sus herramientas de marketing para recopilar "datos de otros sitios de Internet o aplicaciones incluso de personas ajenas a su red, que permiten evaluar el impacto de sus anuncios no sólo en la red social sino fuera de ella" (El País, 2018). Estas declaraciones van de la mano con las denuncias de recopilación de infromación que los usarios generar en Google, WhatsApp y otros medios. Se vislumbra una construcción de una estructura muy pensada en el objetivo del mercado. Por una parte se tiene a sujetos que son observados todo el tiempo, se obtiene todos su datos, sus preferencias, sus anhelos, incluso sus voces; todo esto se procesa y canaliza mediante canales de publicidad que ahora vuelan a las redes y motiven de manera directa a cada uno según sus necesidades, deseos y "búsquedas" en la web, haciendo de la publicidad un negocio muy rentable con pocas fallos en su mecanismo. De esto da cuenta Zuboff (2019) cuando dice que vivimos bajo un capitalismo de vigilancia, donde el comportamiento humano es constantemente vigilado, sus datos son convertidos en producto que se vende, como ella lo dice, el ser humano es materia prima, que se exporta a todo el mundo, a todas las marcas que compran estos datos y crean productos que son fácilmente introducidos en mercados que ya se tiene claro cómo responderán. 
El publicista ahora tiene en sus manos no solo un simple estudio de mercado, no sólo las bondades del producto o servicio que se quiere difundir. Sino que tiene ante sí, la respuesta que tendrá el usuario/consumidor respecto a cualquier producto/servicio que se oferte en el mercado. La gran fábrica de creación de comportamientos humanos, ahora ha engranado con la sociedad de consumo, con el rompimiento de barreras entre lo visual, auditivo de las propagandas y el ser humano. Esta realidad, interpela al publicista y quizá no tiene forma de escapar, no hay lugar para la ética, quizá no hay lugar para una vuelta atrás y dejar a los consumidores/usuarios decidir libremente qué necesitan, qué quiern consumir, sino que constantemente serán un objeto más del deseo de otro.

\section{Conclusión}

La globalización ha desencadenado varios cambios en las sociedades del siglo XXI y el campo de la publicidad no escapa de estas modificaciones. Se ha visto cómo la sociedad de consumo ha permeado todos los campos de la vida y que junto al desarrollo de la tecnología, expresada mediante redes sociales y mass media, han permitido que exista un incremento en la actividad publicitaria. Esto debido a la constante presencia de los personas ante los entornos virtuales y redes sociales, mayormente Facebook, Instagram, Twitter y otras que también son utilizadas no solo como medio para difundir la publicidad y atraer clientes/consumidores, sino que a su vez estas mismas redes son absolutamente capaces de recopilar todo el rastro que deja un usuario. Las aplicaciones se llenan de datos que representan los gustos, intereses y deseos de los internautas que se recopilan mediante las interacciones que se realizan en la web y son transformadas en un producto valioso para el publicista pues ahora tiene todas las herramientas para generar publicidad de manera efectiva.

El artículo encontró que los desafíos que enfrentan los publicistas están muy relacionados con factores que permiten consolidar sistemas o ser parte de una resistencia a la globalización. El rol del publicista puede ayudar a fortalecer la hegemonía de la lengua y pensamiento inglés y mermar el arraigo cultural, o puede ser un factor para el rescate de la cultura local/regional resaltando los valores cultures bajo ataque del proceso unificador de la globalización. Así mismo, puede ser rebelarse ante el abuso y falta de ética de la industria que espía y recopila datos para venderlos o puede ayudar a perpetuar este capitalismo de vigilancia y explotar los recursos obtenidos en favor del consumo global.

El desafío de un mundo cambiante apela no solo a la actualización de conocimientos, técnicas y formación de vanguardia, sino que también debe apelar a la construcción crítica de profesionales que aporten a la construcción de una humanidad y una comunidad valiosa en su diversidad cultural, que cuestione los intereses individuales.

Por otra parte, existen otras campos que le desafían al publicista en el siglo XXI y que son también necesarios investigar; como la función social del publicista, el sistema de educación formal, los perfiles de empresas comunicacionales, etc., esto ayudará al análisis integral de la profesión en este mundo de acelerados cambios.

\section{Referencias}

Baudrillard, J. (1994). The system of collecting. In J. Elsner and R. Cardinal. The cultures of collecting. Harvard University Press

Belk, R. W. (1988). Possessions and the extended self. Journal of Consumer Research, 15, 139-68 
Belk, R. W. (1992). Attachment to possessions. In S. M. Low and I. Altman. Place attachment: Human behavior and environment. Plenum Press.

Belk, R. W., Wallendorf, M., and Sherry, J. (1989). The sacred and the profane in consumer behavior: Theodicy on the odyssey. Journal of Consumer Research, 16(1), 1-38. https://www.jstor.org/stable/2489299

Beltrán y Cruces, R. (2006). Redacción publicitaria. Técnicas para aprender a crear anuncios publicitarios. Trillas \& Eduforma

Benjamin, W. (1968). Unpacking my library - A talk about book collecting. In H. Arendt. Illuminations, Schocken Books.

Bourdieu, P. (1984). Distinction - A social critique of the judgment of taste. Harvard University Press.

Chan, D., \& Kumar, S. (2017). Social Media Strategies for Small and Medium Scale Enterprise in the Klang Valley Region of Malaysia. In N. Ahmad, T. Ramayah, H. Halim, \& S. A. Rahman. (Eds.) Handbook of Research on Small and Medium Enterprises in Developing Countries (pp. 377-400) https:// doi.org/10.4018/978-1-5225-2165-5.cho18

Chetioui, Y., Lebdaoui, H., \& Chetioui, H. (2020). How fashion influencers contribute to consumers' purchase intention. Journal of Fashion Marketing and Management, 24(3), 361-38o. https://doi.org/10.1108/EMJB05-2020-0046

Comaroff, J. (1996). The Empire's old clothes: Fashioning the colonial subject. In D. Howes. Cross-cultural consumption. Routledge.

Cordero-Guti errez, R., \& Lahuerta-Otero, E. (2020). Social media advertising efficiency on higher education programs. Spanish Journal of Marketing - ESIC, 24(2), 247-262. https://doi.org/10.1108/SJME-09-20190075

Cova, B. (1997). Community and consumption: Towards a definition of the linking value of product or services. European Journal of Marketing, 31(3/4), 297-16.

Crammond, R., Omeihe, K. O., Murray, A., \& Ledger, K. (2018). Managing knowledge through social media: Modelling an entrepreneurial approach for Scottish SMEs and beyond. Baltic Journal of Management, 13(3), 303-328. https://doi.org/10.1108/BJM-05-2017-0133

Demont-Heinrich, C. (2008). Beyond Culture and (National) Identity? Language, Globalization and the Discourse of Universal Progress in American Newspaper Coverage of English, Journal of International and Intercultural Communication, 1(2), 136-157, http://doi.org/10.1080/17513050801891960

El País (2018, abril 17). Facebook admite que recopila datos incluso de no usuarios de su red social. El País. https://elpais.com/tecnologia/2018/04/17/actualidad/1523945713_815568.html

Holt, D. B. (1998). Does cultural capital structure American consumption? Journal of Consumer Research, 25, 1-25. https://doi.org/10.1086/209523

Isakova, A. (2021). Impact of globalization and English language on communicative strategies of industrial advertising. IOP Conference Series: Materials Science and Engineering, 1(1100), $012031 \mathrm{https://doi.}$ org/10.1088/1757-899X/1100/1/012031

Jiménez, M.,y Bayón, A. (2021) Facebook gana 24.000 millones en 2020, un 58\% más, y valida su apuesta por el 'ecommerce'. ElPaís. https://cincodias.elpais.com/cincodias/2021/01/27/companias/1611782581_730013. html

Jung, J., Shim, S. W., Jin, H. S., \& Khang, H. (2015). Factors affecting attitudes and behavioural intention towards social networking advertising: a case of Facebook users in South Korea. International Journal of Advertising, 35(2), 248-265. http://doi.org/10.1080/02650487.2015.10147

Kaplan, A. M., \& Haenlein, M. (2010). Users of the world, unite! The challenges and opportunities of Social Media. Business Horizons, 53(1), 59-68. http://doi.org/10.1016/j.bushor.2009.09.00

Kleine, S., Kleine, R., \& Allen, C. (1995). How is a possession "me" or "not me"? Characterizing types and an antecedent of material possession attachment. Journal of Consumer Research, 22(3), 327-44. https:// doi.org/10.1086/209454 
Kozinets, R. V. (1999). E-Tribalized marketing? The strategic implications of virtual communities of consumption. European Management Journal, 17(3), 252-64. https://doi.org/10.1016/So2632373(99)00004-3

Liu, T. (2013). The impact of social networking usage on consumer buying behavior of college students in China. [Conference Paper] International Conference on Advances in Social Science, Humanities, and Management.

Maffesoli, M. (1996). The times of the tribes. Translated by D. Smith. Sage

Rey, J. (2007). Redacción Publicitaria. Técnicas Para Aprender a Crear Anuncios Publicitarios. Questiones Publicitarias, 1(12), 157-60, https://raco.cat/index.php/questionespublicitarias/article/view/v12-rey

Rinallo, D., Maclaran, P., \& Stevens, L. (2016). A Mixed Blessing: Market-mediated Religious Authority in Neopaganism. Journal of Macromarketing, 36(4), 425-442.

Schouten, J. W., \& McAlexander, J. H. (1995). Subcultures of consumption: An ethnography of the new bikers. Journal of Consumer Research, 22, 43-61

Stolz, J., \& Usunier, J-C. (2019) Religions as brands? Religion and spirituality in consumer society. Journal of Management, Spirituality \& Religion, 16(19), 6-31. https://doi.org/10.1080/14766o86.2018.1445008

Waters, R. D., Burnett, E., Lamm, A., \& Lucas, J. (2009). Engaging stakeholders through social networking: How nonprofit organizations are using Facebook. Public Relations Review, 35(2), 102-106 https://doi.org/10.1016/j.pubrev.2009.01.006

Zuboff, S. (2019). The Age for Surveillance Capitalism. The Fight for a Human Future at the New Frontier of Power. Public Affairs

\section{AUTORA}

Diana Anzuátegui Macías. Máster en Relaciones Internacionales y Licenciada en Comunicación Social 